\title{
Off Study Date
}

National Cancer Institute

\section{Source}

National Cancer Institute. Off Study Date. NCI Thesaurus. Code C93610.

The date (and time) when the study subject is removed from the study. 